\title{
Erratum: Matching scalar leptoquarks to the SMEFT at one loop
}

\author{
Valerio Gherardi, ${ }^{a, b}$ David Marzocca ${ }^{b}$ and Elena Venturini ${ }^{c}$ \\ ${ }^{a}$ SISSA, Via Bonomea 265, Trieste 34136, Italy \\ ${ }^{b}$ INFN, Sezione di Trieste, SISSA, \\ Via Bonomea 265, Trieste 34136, Italy \\ ${ }^{c}$ Technische Universität München, Physik-Department, \\ James-Franck-Straße 1, Garching 85748, Germany \\ E-mail: vgherard@sissa.it, david.marzocca@ts.infn.it, \\ elena.venturini@tum.de
}

ERRATUM TO: JHEP07(2020)225

ArXiv EPrint: 2003.12525

The following corrections should be applied to the published version of ref. [1]:

- For the coefficients $\left[C_{q e}\right]^{(1)},\left[C_{\ell u}\right]^{(1)},\left[C_{\ell d}\right]^{(1)},\left[C_{\ell e d q}\right]^{(1)}$, eqs. $(3.84)-(3.87)$, and $\left[G_{q e}\right]^{(1)}$, $\left[G_{\ell u}\right]^{(1)},\left[G_{\ell d}\right]^{(1)},\left[G_{\ell \ell d q}\right]^{(1)}$, eqs. (C.99)-(C.101), all factors $\left(1+L_{i}\right), i=1,3$, should be replaced by $\left(\frac{3}{2}+L_{i}\right)$.

- For the coefficients $\left[C_{\ell q}^{(1)}\right]^{(1)}$ and $\left[C_{\ell q}^{(3)}\right]^{(1)}$, eqs. $(3.77),(3.80)$, and $\left[G_{\ell q}^{(1)}\right]^{(1)},\left[G_{\ell q}^{(3)}\right]^{(1)}$, eqs. (C.96) $-(\mathrm{C} .97)$, the factors $\left(2+\frac{N_{c}^{2}-1}{2 N_{c}}\right) c_{3}^{(5)}=\frac{10}{3} c_{3}^{(5)}$ should be replaced by $\frac{5}{6} c_{3}^{(5)}$.

- For the coefficients $\left[C_{\ell q}^{(1)}\right]^{(1)}$ and $\left[C_{\ell q}^{(3)}\right]^{(1)}$, eqs. $(3.76),(3.79)$, and $\left[G_{\ell q}^{(1)}\right]^{(1)},\left[G_{\ell q}^{(3)}\right]^{(1)}$, eqs. (C.96) $-(\mathrm{C} .97)$, the factor $-\frac{1}{4}\left(\frac{1}{2}+a_{\mathrm{ev}}\right) g^{2}$ should read $+\frac{1}{4}\left(\frac{1}{2}+a_{\mathrm{ev}}\right) g^{2}$.

- In For the coefficients $\left[C_{\ell q}^{(3)}\right]^{(1)}$ and $\left[G_{\ell q}^{(3)}\right]^{(1)}$, eqs. (3.80) and (C.97), the expression $\left(\Lambda_{\ell}^{(31) \dagger}\right)\left(\Lambda_{q}^{(31) \dagger}\right)+\left(\Lambda_{\ell}^{(31)}\right)\left(\Lambda_{q}^{(31)}\right)$ should read $\left(\Lambda_{\ell}^{(31) \dagger}\right)\left(\Lambda_{q}^{(31)}\right)+\left(\Lambda_{\ell}^{(31)}\right)\left(\Lambda_{q}^{(31) \dagger}\right)$ instead.

The correct equations have been substituted in the e-print version of [1].

We thank Konstantinos Mantzaropoulos for pointing out these corrections to us, after a comparison with results obtained via functional methods. 
Open Access. This article is distributed under the terms of the Creative Commons Attribution License (CC-BY 4.0), which permits any use, distribution and reproduction in any medium, provided the original author(s) and source are credited.

\section{References}

[1] V. Gherardi, D. Marzocca and E. Venturini, Matching scalar leptoquarks to the SMEFT at one loop, JHEP 07 (2020) 225 [arXiv:2003.12525] [INSPIRE]. 